CLINICAL REVIEW

\title{
Chronotype and circadian rhythm in bipolar disorder: A systematic review
}

\author{
Matias C.A. Melo ${ }^{a}{ }^{*}$, Rafael L.C. Abreu ${ }^{b}$, Vicente B. Linhares Neto ${ }^{b}$, \\ Pedro F.C. de Bruin ${ }^{a}$, Veralice M.S. de Bruin ${ }^{a}$ \\ a Faculdade de Medicina, Universidade Federal do Ceará, Brazil \\ ${ }^{\mathrm{b}}$ Hospital de Saúde Mental Professor Frota Pinto, Brazil
}

\section{A R T I C L E I N F O}

\section{Article history:}

Received 5 April 2016

Received in revised form

20 June 2016

Accepted 22 June 2016

Available online $\mathrm{xxx}$

\section{Keywords:}

Bipolar disorder

Circadian rhythm

Chronotype

Chronobiology

\begin{abstract}
S U M M A R Y
Despite a complex relationship between mood, sleep and rhythm, the impact of circadian disruptions on bipolar disorder (BD) has not been clarified. The purpose of this systematic review was to define current evidence regarding chronotype and circadian rhythm patterns in BD patients. 42 studies were included, involving 3432 BD patients. Disruption of the biological rhythm was identified, even in drug-naïve BD patients and independently of mood status. Daily profiles of melatonin levels and cortisol indicated a delayed phase. Depression was more frequently associated with circadian alterations than euthymia. Few studies evaluated mania, demonstrating irregular rhythms. Evening type was more common in BD adults. Studies about the influence of chronotype on depressive symptoms showed conflicting results. Only one investigation observed the influences of chronotype in mania, revealing no significant association. Effects of psychoeducation and lithium on rhythm in BD patients were poorly studied, demonstrating no improvement of rhythm parameters. Studies about genetics are incipient. In conclusion, disruption in circadian rhythm and eveningness are common in BD. Prospective research evaluating the impact of circadian disruption on mood symptoms, metabolism, seasonality, the influence of age and the effects of mood stabilizers are needed.
\end{abstract}

๑) 2016 Elsevier Ltd. All rights reserved.

\section{Background}

Mood disorders are multifactorial and heterogeneous conditions influenced by genetic and environmental factors. They are often associated with alterations of sleep and circadian rhythm through a complex and bidirectional relationship [1]. In bipolar disorder (BD), an association between sleep disorders, circadian rhythmicity, chronotype preference, residual mood symptoms and mood episode recurrence is suggested [2].

Circadian rhythmicity and chronotype preference are closely related. Chronotype or morningness-eveningness is the individual preference of the day's period for carrying out activities [3]. It reflects the $24 \mathrm{~h}$ or ultradian propensity for the individual either to be alert or to sleep. Three different chronotypes are identified: morning types, evening types and neither (indifferent) [4].

* Corresponding author. Av. Sargento Hermínio, 880 Quadra A. Bloco 5. Ap 201, Bairro: Monte Castelo, Fortaleza, CE, CEP: 60326-348, Brazil. Tel.: +55 8599631 0299.

E-mail address: matcarv01@yahoo.com.br (M.C.A. Melo).
Circadian rhythmicity describes variability, stability and period functional performance. Actigraphic measures can provide elements of circadian rhythmicity expressed as relative amplitude, interdaily stability and intradaily variability [5].

Behavior alterations of the sleep-wake schedule in relation to the external environment e.g., shift work and jet lag can result in circadian rhythm and sleep-wake disorders potentially altering the alignment of the internal circadian clock with the external environment [6]. Commonly, this rhythm disruption tends to precipitate or exacerbate mood episodes [7]. Furthermore, in animal models, sleep deprivation induces manic episodes [8,9]. It is questioned whether sleep and circadian alterations are triggers for mood symptoms [10].

Conversely, circadian disruptions and sleep complains also can be consequence of mood disorders [11,12]. Manic episodes are frequently characterized by reduced sleep need, while insomnia and hypersomnia occur frequently in depressive phases [13]. Interestingly, sleep loss confers a poor prognosis, increasing the risk of suicide in patients with a suicide attempt history [14]. Even in euthymia, BD patients show more sleep alterations than controls 


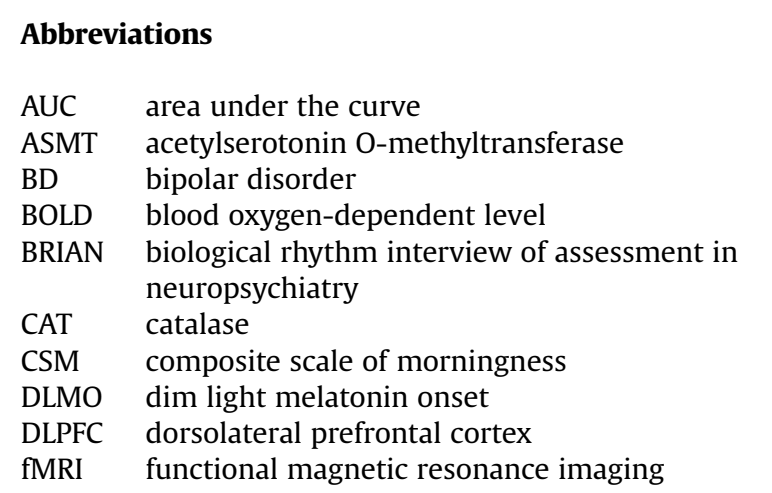

[15]. Given all this evidence, some authors theorize that BD clinical manifestations and pathogenesis can be understood as circadian rhythm alterations [16-18].

Genetic evidence shows a strong relationship between circadian genes and susceptibility to BD [19-21]. Specific gene variants link to different kinds of mood disorders. Two polymorphisms of CLOCK (rs1801260 and rs11932595) relate with BD-II ${ }^{1}$; and two of TIM (rs2291739, rs11171856), with unipolar depression [22]. Furthermore, circadian clock components can influence the response to mood stabilizers. Some circadian clock components, e.g., Per2, Cry 1 and $\operatorname{Rev}$-erb $\alpha$, probably increase individual sensibility to the therapeutic effects of lithium $[23,24]$.

Chronotherapeutic interventions have been successfully used in BD. Sleep deprivation combined with intensive light therapy is a good adjunct to standard treatments for depressive episodes [25]. The blue-blocking regime in mania, a virtual darkness therapy, was followed by a rapid and sustained decline in mood symptoms accompanied by a reduction in total sleep, a reduction in motor activity during sleep intervals, and an increased regularity of sleep intervals [26]. Interpersonal and social rhythm therapy potentially improves mood symptoms and prevents relapse of mood episodes [27]. A recent metanalysis involving $489 \mathrm{BD}$ patients showed that light therapy reduces disease severity [28]. All these findings indicate the importance of studying the relationship between sleep, circadian rhythm and mood.

Previous evidence shows that different physiological aspects are associated with chronotypes. It is linked to both positive and negative affects, and to specific clinical conditions e.g., metabolic and sleep disorders [29-33]. Eveningness is associated with many mental disorders [34]. It is a probable independent risk factor related to severity, suicidal ideation, nonremission and poor response to treatment in depressive episodes [35-38]. Despite all this evidence, clinical repercussions of circadian rhythm alterations and chronotype preferences in BD patients need more clarification.

The objective of this study is to perform a systematic review to define current evidence about chronotype and circadian rhythm patterns in patients with bipolar disorder.

\section{Methods}

\section{Search strategies}

This review focuses on circadian rhythm and chronotype. Measures included behavior questionnaires, actigraphy, genetic

\footnotetext{
1 Bipolar disorder can be classified into type I and II. BD-I is characterized by at least one manic episode, while BD-II is marked by at least one episode of hypomania and at least one episode of major depression
}

GSS

GST

L5

M10

MCTQ Munich chronotype questionnaire

MDA malondialdehyde

MDD major depressive disorder

MEQ morningness-eveningness questionnaire

MRI magnetic resonance imaging

SAD seasonal affective disorders

SRM social rhythm metric

SOD superoxide dismutase and hormonal evaluation. Two researchers performed an electronic search of PubMed, Cochrane Library and ClinicalTrials.gov. Keywords used were 'chronotype' or 'circadian rhythm' or 'biological rhythm' or 'sleep' AND 'bipolar disorder' or 'mania' or 'bipolar depression'. Manual searches were also conducted, using reference lists from identified articles.

We included all articles published in the last 20 y (1995-2015) evaluating chronotype and circadian variations in BD patients. Reviews, case reports, conference abstracts, expert opinions, animal experiments and incomplete clinical trials were excluded. Articles duplicated or unavailable in the English language were removed. Research with patients with mental disorders that did not analyze bipolar disorder and other mental diseases separately was not considered. Articles that reported only sleep patterns or sleep disorders but not chronotype or circadian variations were excluded. Genetic studies were limited to those that identified relations between genes and their repercussions on biological rhythm. Interventional studies that evaluated the impact on circadian functioning were included. Articles measuring levels of hormones such as melatonin or cortisol and their daily hormonal profile were selected.

\section{Sleep and rhythm measures}

Behavior questionnaires and/or actigraphy assessed sleep and rhythm providing information about individual differences and their relationship with other biological functions. The morningness eveningness questionnaire (MEQ) is an instrument with good reliability and stability, used to describe chronotype or phase preference [39]. The circadian type questionnaire investigates the amplitude and stability of circadian rhythm $[40,41]$. The composite scale of morningness (CSM) consists of 13 items from two different scales: MEQ and diurnal type scale. It assesses individual differences in the time of day that a person prefers to carry out various activities, and classifies people as morning-type or evening-type [42]. The CSM demonstrated good test-retest reliability and adequate external validity [43]. The social rhythm metric (SRM) is a self-report instrument designed to quantify social rhythm regularity. It helps to structure the day cognitively and to plan possible changes routines. The Munich chronotype questionnaire (MCTQ), a recent instrument used to determine the circadian type, is a good indicator of melatonin onset; the MCTQ is calculated by the midpoint of sleep between onset and offset on days off from work [42]. The biological rhythm interview of assessment in neuropsychiatry (BRIAN) consists of 18 items evaluating five domains: sleep, activity, sociality, eating habits and rhythm and it refers to the last 14 d [44].

Circadian rhythm analysis can be yielded by actigraphy. Several components of circadian rhythm i.e., as relative amplitude, 
interdaily stability and intradaily variability are provided. It is a good instrument for estimating sleep length and fragmentation in bipolar disorder, revealing a good correlation with polysomnography [45]. Measures such as the Least5 (L5) and Most10 (M10) indicate the regularity of sleep, activity or inactivity. The L5 provides the average activity level for the sequence of the least five active hours indicating how restful (inactive) and regular the sleep periods are. The M10 average provides the average activity level for the sequence of the highest (most) 10 active hours. The onset of L5 (restful hours) and M10 (active hours) gives an indication of the phase preference or chronotype. Among BD patients in euthymia, comparisons between objective and subjective sleep and circadian markers suggest that CSM has a good association with M10 onset and L5 onset measured in actigraphy $[5,40]$.

Hormonal levels such as melatonin or cortisol, determined throughout the day, can express endogenous circadian rhythm. However, isolated measures have lower reliability as a tool to indicate circadian rhythm [46]. In this review, these cases were not included.

\section{Data analysis}

All articles were displayed on a table in chronological sequence with the following data: names of authors, publication year, study design, participants, mood status, sleep and rhythm measures and main results. The results were organized, considering the following topics: 1) disruption of biological rhythm - 19 studies (45.2\%); 2) chronotype - 15 studies (35.7\%); 3) circadian rhythm and biomarkers - six studies (14.3\%); 4) genetics - six studies (14.3\%).

\section{Results}

\section{Study selection}

The initial electronic database search from 1995 to 2015 resulted in 1654 hits. Additionally, four records were found in the reference list of identified studies. Initially, 1658 articles were included. After careful examination, 1514 were excluded: 509 were published before 1995; 479 focused on other conditions; 236 were reviews, conference abstracts, and expert's opinions; 143 were duplicate; 51 animal experiments; 42 case reports; 21 incomplete studies, and
31 did not analyze bipolar disorder and other mental diseases separately; and two were unavailable in English language. Thus, 144 articles were selected about sleep and rhythm in bipolar disorder.

Thereafter, 102 articles were removed because referred to other topics: 65 concerned only sleep parameters; 12 only people at high risk for bipolar disorder (not bipolar patients); two compared objective and subjective sleep measures; and 23 focused on a population with multiple psychiatric disorders. 42 studies were finally selected (Fig. 1).

\section{Study description}

Overall, 3432 BD patients were finally included in this review. Table 1 shows data in the following order: author and year, study design, participants (number), mood status, other characteristics (gender, age, etc.), main outcomes (sleep, rhythm, etc.) and summary results. Number of patients per study varied from 8 to 260 . Studies were mostly cross-sectional and case control.

Two intervention studies were found. One randomized clinical trial (45 patients) evidenced no significant impact of psychoeducation on biological rhythms in BD [47]. Another study (29 patients) registered melatonin levels before and after exposure to light. Euthymic BD patients showed lower melatonin levels on the light night, at baseline and following light exposure and a later peak time for melatonin on the dark night [48].

Two prospective cohorts were identified. Seleem et al., 2015 (257 patients) demonstrated that evening preference is a chronic characteristic in BD patients. In this study, evening preference was not associated with polarity type, or mood state in BD, suggesting that this characteristic may be a trait marker [49]. Shen et al., 2008 (206 patients) suggested that circadian irregularity is a prognostic factor related to shorter euthymia periods. Survival analyses indicated that both diagnosis and social rhythm regularity significantly predicted the time to participants' first prospective onset of major depressive, hypomanic and manic episodes [50].

The majority of studies - 23 studies including 2798 patients $(81.5 \%)$ - were based only on subjective measures. Most used scales were composite scale of morningness (CSM) - nine studies involving 1556 patients (45.3\%); biological rhythm interview of assessment in neuropsychiatry (BRIAN) - six studies, 593 patients

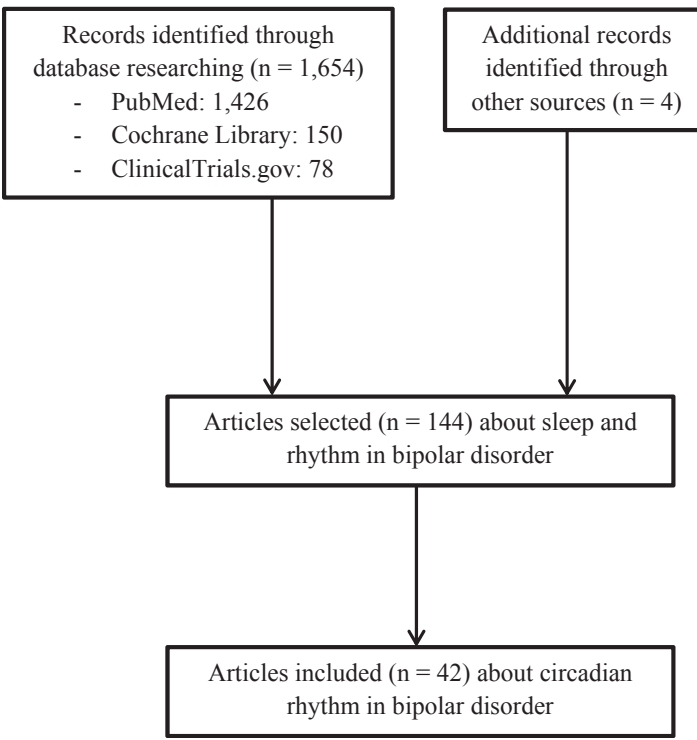

1,514 excluded:

- 509 published before 1995 ;

- 479 focused on other subjects;

236 reviews, conference abstracts, and expert opinions;

143 duplicated;

51 referred to animal experiments;

- 42 case reports;

- 31 did not analyze bipolar disorder and other mental diseases separately;

21 incomplete studies;

- 2 unavailable in English language.

102 excluded:

65 focused only on sleep parameters and not on circadian rhythm;

23 focused on other subjects;

- 12 referred to people at high risk for bipolar disorder (not bipolar patients);

2 only compared objective and subjective sleep measures.

Fig. 1. Flowchart for study selection. 


\begin{tabular}{|c|c|c|c|c|c|c|}
\hline $\begin{array}{l}\text { Authors, year } \\
\text { of publication }\end{array}$ & Study design & Participants & Mood status & $\begin{array}{l}\text { Other } \\
\text { characteristics }\end{array}$ & $\begin{array}{l}\text { Sleep and } \\
\text { rhythm } \\
\text { measures }\end{array}$ & Main results \\
\hline $\begin{array}{l}\text { Ashman et al., } 1999 \\
\text { [53] }\end{array}$ & $\begin{array}{l}\text { Case-control } \\
\text { study }\end{array}$ & $\begin{array}{l}9 \text { BD and } 9 \\
\text { controls }\end{array}$ & Any & $\begin{array}{l}\text { Rapid cycling. } \\
\text { Age } 29-61 \text { y. }\end{array}$ & SRM & $\begin{array}{l}\text { Patients had lower activity levels and more phase delay. } \\
\text { Depression phase was associated with more phase } \\
\text { delay than euthymia and hypomania. }\end{array}$ \\
\hline $\begin{array}{l}\text { Nurnberger et al., } \\
2000[48]\end{array}$ & $\begin{array}{l}\text { Intervention } \\
\text { study }\end{array}$ & $\begin{array}{l}29 \mathrm{BD}, 24 \mathrm{MDD} \\
\text { and } 50 \text { controls }\end{array}$ & Euthymia & Age $22-63$ y. & $\begin{array}{l}\text { Melatonin } \\
\text { levels }\end{array}$ & $\begin{array}{l}\text { BD patients were exposed to a } 500 \text {-lux light and showed } \\
\text { lower melatonin levels on the light night and later peak } \\
\text { time for melatonin on the dark night. }\end{array}$ \\
\hline $\begin{array}{l}\text { Hakkarainen et al., } \\
2003[76]\end{array}$ & $\begin{array}{l}\text { Retrospective } \\
\text { cohort }\end{array}$ & $\begin{array}{l}39 \mathrm{BD}, 8 \text { non- } \\
\text { BD and } 20 \\
\text { controls }\end{array}$ & Any & $\begin{array}{l}\text { Twins. Age } 29 \\
-57 \mathrm{y} \text {. }\end{array}$ & SPAQ and MEQ & $\begin{array}{l}\text { No significant differences in morningness-eveningness } \\
\text { between BD and controls. Preference for evening was } \\
\text { associated with a higher global seasonality score. BD } \\
\text { slept longer, compared with controls. }\end{array}$ \\
\hline $\begin{array}{l}\text { Jones et al., } 2005 \\
\text { [57] }\end{array}$ & $\begin{array}{l}\text { Case-control } \\
\text { study }\end{array}$ & $\begin{array}{l}19 \mathrm{BD} \text { and } 19 \\
\text { controls }\end{array}$ & Euthymia & $\begin{array}{l}\text { F/M: 2.8. Mean } \\
\text { age } 44.37 \mathrm{y} .\end{array}$ & $\begin{array}{l}\text { SRM and } \\
\text { actigraphy for } 7 \\
\text { days }\end{array}$ & $\begin{array}{l}\text { BD had less stable and more variable circadian activity } \\
\text { patterns than controls and more intradaily variability. } \\
\text { No differences found on objective sleep measures. }\end{array}$ \\
\hline $\begin{array}{l}\text { Mansour et al., } \\
2005 \text { [75] }\end{array}$ & $\begin{array}{l}\text { Case-control } \\
\text { study }\end{array}$ & $\begin{array}{l}75 \mathrm{BD}, 81 \mathrm{Sz} / \\
\text { SzA, and } 349 \\
\text { controls }\end{array}$ & Any & No-specified & $\operatorname{CSM}$ & $\begin{array}{l}\text { BD showed different rhythm from controls: lower CSM } \\
\text { scores. Different from other groups, CSM scores were } \\
\text { distributed bimodally among BD: Rapid cycling BD were } \\
\text { more likely to have lower CSM scores. }\end{array}$ \\
\hline $\begin{array}{l}\text { Benedetti et al., } \\
2007 \text { [66] }\end{array}$ & Cross-sectional & $39 \mathrm{BD}$ & $\begin{array}{l}\text { Depression } \\
\text { without } \\
\text { psychosis }\end{array}$ & M/F: 1.05 & $\begin{array}{l}\text { Actigraphy for } \\
48 \mathrm{~h}\end{array}$ & $\begin{array}{l}\text { CLOCK variant showed a significantly higher evening } \\
\text { activity and a reduced amount of sleep during the night. } \\
\text { Lithium-treated patients had higher activity levels in the } \\
\text { evening and a trend toward a later morning awakening. }\end{array}$ \\
\hline $\begin{array}{l}\text { Ahn et al., } 2008 \\
\text { [68] }\end{array}$ & $\begin{array}{l}\text { Case-control } \\
\text { study }\end{array}$ & $\begin{array}{l}92 \mathrm{BD}, 113 \mathrm{Sz} \text {, } \\
\text { and } 95 \text { controls }\end{array}$ & Euthymia & $\begin{array}{l}\text { F/M: 1.1. Mean } \\
\text { age } 34.3 \mathrm{y} \text {. }\end{array}$ & CSM & $\begin{array}{l}\text { BD had lower CSM scores and greater preference for } \\
\text { evening activity and late sleep timing than controls. Sz } \\
\text { patients did not show different patterns in circadian } \\
\text { preference. }\end{array}$ \\
\hline $\begin{array}{l}\text { Salvatore et al., } \\
\quad 2008 \text { [61] }\end{array}$ & $\begin{array}{l}\text { Case-control } \\
\text { study }\end{array}$ & $\begin{array}{l}36 \text { manic/ } \\
\text { mixed } \mathrm{BD} \text {, and } \\
32 \text { controls }\end{array}$ & $\begin{array}{l}\text { Manic, mixed } \\
\text { status and } \\
\text { euthymia }\end{array}$ & $\begin{array}{l}\mathrm{F} / \mathrm{M}=4.15 \\
\text { Mean age: } \\
44.4 \text { y. }\end{array}$ & $\begin{array}{l}\text { Actigraphy for } \\
72 \mathrm{~h}\end{array}$ & $\begin{array}{l}\text { Both manic/mixed and recovered BD had acrophase } \\
\text { advance and lower daily activity Euthymic BD had } \\
\text { lower mean of } 24 \text {-h motor activity and higher total } \\
\text { sleep. }\end{array}$ \\
\hline $\begin{array}{l}\text { Shen et al., } 2008 \\
\quad[50]\end{array}$ & $\begin{array}{l}\text { Cohort study } \\
(\sim 33 \mathrm{mo})\end{array}$ & $\begin{array}{l}206 \text { BD and } 208 \\
\text { controls }\end{array}$ & Not specified & $\begin{array}{l}\text { F/M: } 1.43 . \text { Age } \\
18-24 \text { y. }\end{array}$ & SRM & $\begin{array}{l}\text { BD reported fewer regular daily activities. Less social } \\
\text { rhythm regularity predicted a shorter time to onset of } \\
\text { affective episode. }\end{array}$ \\
\hline $\begin{array}{l}\text { Kripke et al., } 2009 \\
\text { [85] }\end{array}$ & $\begin{array}{l}\text { Case-control } \\
\text { study }\end{array}$ & $\begin{array}{l}130 \mathrm{BD} \text { and } 561 \\
\text { relatives }\end{array}$ & Not specified & No-specified & $\begin{array}{l}\text { Basic language } \\
\text { morningness }\end{array}$ & $\begin{array}{l}\text { Polymorphisms of PER } 3 \text { and CSNK1E were associated } \\
\text { with greater eveningness in BD patients. }\end{array}$ \\
\hline
\end{tabular}

Soreca et al., 2009 [78]

Cross-sectional

$29 \mathrm{BD}$

BALM) scale

Wood et al., 2009

Case-control

Euthymia

$190 \mathrm{BD}$ and 128

Any

[70]

study

controls

Giglio et al., 2010

[71]

study

$81 \mathrm{BD}$ and 79

controls

Euthymia

$\mathrm{M}=2.57$

Mean age

$43.5 \mathrm{y}$.

$260 \mathrm{BD}$ and 350

controls

study

Minassian et al.,

2010 [63]
Case-control study
$28 \mathrm{BD}, 17 \mathrm{Sz}$, and 21 controls
Mean age

$35.5 \mathrm{y}$.

Mean age
$\mathrm{F} / \mathrm{M}=1.36 . \mathrm{Ag}$

CSM and PSQI

$\mathrm{F} / \mathrm{M}=4.13$

Mean age

Evening preference was associated with higher percentage of body fat.

BD patients are more likely to be evening types. CSM scores for BD-I and BD-II were different from the controls. High depressive mood scores are more likely to be associated with evening type.

BD had more evening preference. Subthreshold depressive and manic symptoms were unrelated to chronotype in patients. Circadian preference was associated only with sleep latency and there was no association with other PSQI subscales

$\mathrm{F} / \mathrm{M}=1.34 . \quad \mathrm{CSM}$

$\mathrm{M} / \mathrm{F}=1.15$.

$34.1 \mathrm{y}$.
CLOCK 3111T/C showed a genotypic association with bipolar disorder. C allele carriers $(\mathrm{C} / \mathrm{C}$ and $\mathrm{C} / \mathrm{T}$ genotypes) were more associated with eveningness. Manic BD had higher levels of motor activity than Sz. Motor activity showed a modest relationship with symptom ratings of mania and psychosis.
PSQI and

rhythms

CSM

LifeShirt, a monitoring device in the form of a

wearable

upperbody

garment 


\begin{tabular}{|c|c|c|c|c|c|}
\hline $\begin{array}{l}\text { Brambilla et al., } \\
2012 \text { [77] }\end{array}$ & $\begin{array}{l}\text { Cross-sectional } \\
\text { study }\end{array}$ & $\begin{array}{l}67 \mathrm{BD} \text { and } 46 \\
\text { remitted MDD }\end{array}$ & Euthymia & $\begin{array}{l}\mathrm{F} / \mathrm{M}=2.42 . \\
\text { Mean age } \\
51.32 \mathrm{y} .\end{array}$ & $\begin{array}{l}\text { SPAQ MEQ and } \\
\text { medical } \\
\text { outcomes study } \\
\text { sleep scale }\end{array}$ \\
\hline $\begin{array}{l}\text { Chung et al., } 2012 \\
\text { [74] }\end{array}$ & $\begin{array}{l}\text { Cross-sectional } \\
\text { study }\end{array}$ & $\begin{array}{l}106 \text { BD-I, } 43 \\
\text { BD-II, and } 108 \\
\text { MDD }\end{array}$ & Not specified & $\begin{array}{l}\mathrm{F} / \mathrm{M}=2.34 \\
\text { Mean age: } 38 \mathrm{y} \\
\text { (type I) and } \\
32 \mathrm{y}(\mathrm{II}) .\end{array}$ & CSM \\
\hline $\begin{array}{l}\text { Faurholt-Jepsen } \\
\text { et al., } 2012 \text { [67] }\end{array}$ & $\begin{array}{l}\text { Case-control } \\
\text { study }\end{array}$ & $\begin{array}{l}18 \mathrm{BD}, 20 \mathrm{MDD} \text {, } \\
\text { and } 31 \text { controls }\end{array}$ & $\begin{array}{l}\text { Euthymia or } \\
\text { depression }\end{array}$ & $\begin{array}{l}\mathrm{F} / \mathrm{M}=1.56 . \text { Age } \\
18-60 \mathrm{y}\end{array}$ & $\begin{array}{l}\text { Combined } \\
\text { heart rate and } \\
\text { movement } \\
\text { sensor }\end{array}$ \\
\hline $\begin{array}{l}\text { van der Werf- } \\
\text { Eldering et al., } \\
2012 \text { [81] }\end{array}$ & $\begin{array}{l}\text { Cross-sectional } \\
\text { study }\end{array}$ & $65 \mathrm{BD}$ & $\begin{array}{l}\text { Euthymia or } \\
\text { depression }\end{array}$ & $\begin{array}{l}\mathrm{F} / \mathrm{M}=2.1 . \text { Age } \\
18-65 \mathrm{y} .\end{array}$ & $\begin{array}{l}\text { Diurnal cortisol } \\
\text { levels and } \\
\text { cortisol } \\
\text { suppression } \\
\text { test. }\end{array}$ \\
\hline $\begin{array}{l}\text { Boudebesse et al., } \\
2013 \text { [41] }\end{array}$ & $\begin{array}{l}\text { Cross-sectional } \\
\text { study }\end{array}$ & $\begin{array}{l}140 \mathrm{BD} \text { and } 156 \\
\text { controls }\end{array}$ & Euthymia & $\begin{array}{l}\mathrm{F} / \mathrm{M}=1.05 \\
\text { Mean age: } \\
41.57 \mathrm{y} .\end{array}$ & CTI and CSM \\
\hline $\begin{array}{l}\text { Robillard et al., } \\
2013 \text { [79] }\end{array}$ & $\begin{array}{l}\text { Case-control } \\
\text { study }\end{array}$ & $\begin{array}{l}18 \mathrm{BD} \text { and } 14 \\
\text { MDD }\end{array}$ & Depression & $\begin{array}{l}\mathrm{F} / \mathrm{M}=3 . \text { Age } 15 \\
-30 \mathrm{y}\end{array}$ & $\begin{array}{l}\text { Actigraphy }-7 \\
\text { days and } \\
\text { melatonin } \\
\text { levels }\end{array}$ \\
\hline $\begin{array}{l}\text { Rosa et al., } 2013 \\
\text { [65] }\end{array}$ & $\begin{array}{l}\text { Case-control } \\
\text { study }\end{array}$ & $\begin{array}{l}107 \mathrm{BD} \text { and } 100 \\
\text { controls }\end{array}$ & Euthymia & $\begin{array}{l}\mathrm{M} / \mathrm{F}=1.28 \\
\text { Mean age } 43 \mathrm{y}\end{array}$ & BRIAN \\
\hline $\begin{array}{l}\text { Saunders et al., } \\
2013 \text { [73] }\end{array}$ & $\begin{array}{l}\text { Retrospective } \\
\text { cohort study }\end{array}$ & $\begin{array}{l}119 \mathrm{BD} \text { and } 136 \\
\text { controls }\end{array}$ & Euthymia & $\begin{array}{l}\mathrm{F} / \mathrm{M}=2 . \text { Mean } \\
\text { age } 41 \mathrm{y} .\end{array}$ & $\begin{array}{l}\text { PSQI, ESS and } \\
\text { MCTQ }\end{array}$ \\
\hline $\begin{array}{l}\text { Baek et al., } 2014 \\
\text { [69] }\end{array}$ & $\begin{array}{l}\text { Case-control } \\
\text { study }\end{array}$ & $\begin{array}{l}200 \mathrm{BD} \text { and } 270 \\
\text { controls }\end{array}$ & Euthymia & $\begin{array}{l}\mathrm{F} / \mathrm{M}=1.9 . \text { Age } \\
18-45 \mathrm{y} .\end{array}$ & $\begin{array}{l}\text { CSM, STQ and } \\
\text { SPAQ }\end{array}$ \\
\hline $\begin{array}{l}\text { Cudney et al., } 2014 \\
\text { [51] }\end{array}$ & $\begin{array}{l}\text { Case-control } \\
\text { study }\end{array}$ & $\begin{array}{l}52 \mathrm{BD} \text { and } 30 \\
\text { controls }\end{array}$ & $\begin{array}{l}\text { Euthymia and } \\
\text { depression }\end{array}$ & $\begin{array}{l}\text { All females. } \\
\text { Mean age } \\
40.75 \mathrm{y} .\end{array}$ & BRIAN \\
\hline $\begin{array}{l}\text { Etain et al., } 2014 \\
\text { [19] }\end{array}$ & $\begin{array}{l}\text { Case-control } \\
\text { study }\end{array}$ & $\begin{array}{l}239 \mathrm{BD} \text { and } 873 \\
\text { controls }\end{array}$ & Euthymia & $\mathrm{M} / \mathrm{F}=1.3$ & CSM and CTI \\
\hline $\begin{array}{l}\text { Faria et al., } 2014 \\
\quad[47]\end{array}$ & $\begin{array}{l}\text { Randomized } \\
\text { clinical trial }\end{array}$ & $45 \mathrm{BD}$ & Any & Mean age 24 y. & BRIAN \\
\hline $\begin{array}{l}\text { Geoffroy et al., } 2014 \\
\text { [62] }\end{array}$ & $\begin{array}{l}\text { Case-control } \\
\text { study }\end{array}$ & $\begin{array}{l}25 \mathrm{BD} \text { and } 28 \\
\text { controls }\end{array}$ & Euthymia & $\begin{array}{l}\mathrm{F} / \mathrm{M}=1.2 . \\
\text { Mean age } \\
53.50 \mathrm{y} .\end{array}$ & $\begin{array}{l}\text { PSQI and } \\
\text { actigraphy }\end{array}$ \\
\hline
\end{tabular}

MDD had greater sleep disturbance than BD. Morningness type had less disturbed and more adequate sleep quality. A family history for mood disorders was associated with higher fluctuations throughout seasons.

No difference in CSM total score among the three groups. BD-I had a higher score of evening tiredness than BD-II. BD-I had a higher mean score than MDD in morning alertness.

$\mathrm{BD}$ and MDD had higher sleeping heart rates than controls. Fitness, acceleration and activity energy expenditure were lower in unipolar patients, whereas there was no significant difference between BD and controls.
cho significant difference between BD and

An association between depressive symptoms and cognitive impairment was found. Correlations betwee HPA axis activity and cognitive functioning or depressive symptoms were not identified.

BD patients were more languid and showed an evenin preference, but they did not differ from the controls with regard to flexibility/rigidity.

Evening melatonin onset was reduced and delayed in a great proportion of young people with mood disorders, and these abnormalities were more prominent in those with bipolar as compared to unipolar depression.

$\mathrm{BD}$ experienced greater biological rhythm alterations and more impaired sleep/social and activity domains. Correlation was found between biological rhythms with residual depressive symptoms and functioning

Chronotype did not differ between two groups. BD had poorer sleep quality in PSQI and its subscales: sleep latency, subjective quality, sleep disturbance, sleep medication, and daytime dysfunction. BD also had more medication,
sleepiness

BD-I and BD-II had lower CSM scores, higher global seasonality scores and more SAD as compared to controls. BD-II had lower CSM scores than BD-I. Patients with $\mathrm{BD}$ and SAD had more evening preference. BRIAN scores showed a greater circadian rhythm disruption. Circadian rhythms disruption and number of psychiatric medications were independent predictors of lipid damage and malondialdehyde levels in BD.

Polymorphisms rs774045 was associated with

eveningness; and rs782931 was correlated with rigid circadian type.

One group received psychoeducation and medication (combined intervention); and the other, only

medication (standard intervention). Both groups had remission of depressive symptoms, but none showed reduction of manic symptoms. No influence of psychoeducation on rhythm. Only the standar intervention group improved the BRIAN domains: sleep. activity, social rhythm and total score.

An association between the GG genotype, longer sleep duration ( $p=0.03)$, greater activity $(\mathrm{p}=0.015)$ and a higher interday stability ( $\mathrm{p}=0.003$ ) was shown.

(continued on next page) 


\begin{tabular}{|c|c|c|c|c|c|c|}
\hline $\begin{array}{l}\text { Authors, year } \\
\text { of publication }\end{array}$ & Study design & Participants & Mood status & $\begin{array}{l}\text { Other } \\
\text { characteristics }\end{array}$ & $\begin{array}{l}\text { Sleep and } \\
\text { rhythm } \\
\text { measures }\end{array}$ & Main results \\
\hline $\begin{array}{l}\text { Gonzalez et al., } \\
2014 \text { [56] }\end{array}$ & $\begin{array}{l}\text { Cross-sectional } \\
\text { study }\end{array}$ & $42 \mathrm{BD}$ & Any & $\begin{array}{l}\mathrm{F} / \mathrm{M}=1.8 . \text { Age } \\
\text { mean } 53.50 \mathrm{y} .\end{array}$ & $\begin{array}{l}\text { Actigraphy - } 7 \\
\text { days }\end{array}$ & $\begin{array}{l}\text { Greater severity of manic symptoms correlated with a } \\
\text { lower degree of rhythmicity and less robust rhythms. } \\
\text { No relationship was noted between the degree of } \\
\text { depression and 24-h autocorrelation scores or circadian } \\
\text { quotient }\end{array}$ \\
\hline $\begin{array}{l}\text { Kim et al., } 2014 \\
\text { [72] }\end{array}$ & $\begin{array}{l}\text { Case-control } \\
\text { study }\end{array}$ & $\begin{array}{l}30 \text { BD teens and } \\
45 \text { controls }\end{array}$ & Any & Age $7-17$ y. & MESC & $\begin{array}{l}\text { BD teens reported greater eveningness. No difference } \\
\text { between two groups in latency age }(<13 \text { y old })\end{array}$ \\
\hline $\begin{array}{l}\text { Krane-Gartiser } \\
\text { et al., } 2014 \text { [64] }\end{array}$ & $\begin{array}{l}\text { Case-control } \\
\text { study }\end{array}$ & $\begin{array}{l}18 \text { BD with } \\
\text { mania, } 12 \mathrm{BD} \\
\text { with } \\
\text { depression and } \\
28 \text { controls }\end{array}$ & $\begin{array}{l}\text { Mania and } \\
\text { Depression }\end{array}$ & $\begin{array}{l}\text { Hospitalized } \\
\text { patients. F/ } \\
\mathrm{M}=1.5\end{array}$ & $\begin{array}{l}\text { Actigraphy - } \\
24 \mathrm{~h}\end{array}$ & $\begin{array}{l}\text { BD with depression had a lower mean activity level, but } \\
\text { higher variability. The motor activity was lower in } \\
\text { mania and depression in the morning ( }(\mathrm{p}<0.001) \text {, but } \\
\text { not in evening. In depression, BD had lower activity and } \\
\text { higher variability than mania and controls. }\end{array}$ \\
\hline $\begin{array}{l}\text { Kripke et al., } 2014 \\
{[84]}\end{array}$ & $\begin{array}{l}\text { Case-control } \\
\text { study }\end{array}$ & $\begin{array}{l}8 \mathrm{BD} \text { and } 37 \\
\text { controls }\end{array}$ & Not specified & Not specified & $\begin{array}{l}\text { MEQ and } \\
\text { actigraphy - } \\
14 \text { days }\end{array}$ & $\begin{array}{l}\text { Delayed sleep and "eveningness" were inversely } \\
\text { associated with loci in circadian genes (rs2482705) and } \\
\text { RORC (rs3828057). A group of haplotypes overlapping } \\
\text { BHLHE40 was associated with non-24-h sleep-wake } \\
\text { cycles, and less robustly, with delayed sleep and bipolar } \\
\text { disorder. }\end{array}$ \\
\hline $\begin{array}{l}\text { McKenna et al., } \\
2014 \text { [58] }\end{array}$ & $\begin{array}{l}\text { Case-control } \\
\text { study }\end{array}$ & $\begin{array}{l}14 \mathrm{BD} \text { and } 14 \\
\text { controls }\end{array}$ & Euthymia & Age $30-79 \mathrm{y}$. & $\begin{array}{l}\text { Actigraphy - } \\
24 \mathrm{~h}\end{array}$ & $\begin{array}{l}\text { BD had less efficient sleep, less activity, less robust } \\
\text { rhythm, and a smaller amplitude-to-width ratio. } \\
\text { Variability in sleep/circadian rhythm was associated } \\
\text { with degree of abnormality of brain response in the } \\
\text { dorsolateral prefrontal cortex and supramarginal gyri } \\
\text { brain response on a working memory task. }\end{array}$ \\
\hline $\begin{array}{l}\text { Rock et al., } 2014 \\
\text { [60] }\end{array}$ & $\begin{array}{l}\text { Case-control } \\
\text { study }\end{array}$ & $\begin{array}{l}19 \mathrm{BD} \text { and } 21 \\
\text { controls }\end{array}$ & Euthymia & $\begin{array}{l}\mathrm{F} / \mathrm{M}=1.1 \\
\text { Mean age } \\
20.1 \mathrm{y} .\end{array}$ & $\begin{array}{l}\text { MEQ and } \\
\text { actigraphy - } \\
14 \text { days }\end{array}$ & $\begin{array}{l}\text { BD had increased movement during sleep, as assessed } \\
\text { by the fragmentation index. BD had lower circadian } \\
\text { amplitude and greater activity levels during their least } \\
\text { active sleep phase }(02: 00-07: 00 \mathrm{~h}) \text {. }\end{array}$ \\
\hline $\begin{array}{l}\text { Duarte Faria et al., } \\
2015 \text { [52] }\end{array}$ & $\begin{array}{l}\text { Case-control } \\
\text { study }\end{array}$ & $\begin{array}{l}49 \text { drug-naive } \\
\text { BD, } 74 \text { MDD, } \\
\text { and } 94 \text { controls }\end{array}$ & Any & $\begin{array}{l}\text { F/M: } 3.8 \text {. Mean } \\
\text { age } 21.88\end{array}$ & BRIAN & $\begin{array}{l}\text { Disruption in biological rhythm was higher in BD than } \\
\text { MDD and controls. Biological rhythm was associated } \\
\text { with bipolar disorder independently of current mood } \\
\text { state, differently of MDD. }\end{array}$ \\
\hline $\begin{array}{l}\text { Nováková et al., } \\
2015 \text { [80] }\end{array}$ & $\begin{array}{l}\text { Case-control } \\
\text { study }\end{array}$ & $\begin{array}{l}60 \mathrm{BD} \text { in mania, } \\
22 \text { in } \\
\text { depression, and } \\
19 \text { controls }\end{array}$ & $\begin{array}{l}\text { Mania and } \\
\text { Depression }\end{array}$ & $\begin{array}{l}\text { Mean age } \\
44.5 \mathrm{y} \text { in } \\
\text { depression, and } \\
40.4 \mathrm{y} \text { in mania. }\end{array}$ & $\begin{array}{l}\text { Daily profiles of } \\
\text { melatonin } \\
\text { levels }\end{array}$ & $\begin{array}{l}\text { Melatonin higher during daytime in mania phase; the } \\
\text { Per1and Nr1d1 profiles were advanced in mania } \\
\text { compared with depression phase. The amplitude of the } \\
\text { Nr1d1 expression profile was higher in mania than in } \\
\text { depression. }\end{array}$ \\
\hline $\begin{array}{l}\text { Pinho et al., } 2015 \\
\text { [54] }\end{array}$ & $\begin{array}{l}\text { Case-control } \\
\text { study }\end{array}$ & $\begin{array}{l}260 \text { BD and } 191 \\
\text { controls }\end{array}$ & Any & $\begin{array}{l}\text { F/M: 3.8. Mean } \\
\text { age } 21.9 \text { y. }\end{array}$ & BRIAN & $\begin{array}{l}\text { BD had more biological rhythms disruption, an } \\
\text { independent predictor of poor functioning. Depressed } \\
\text { patients had greater biological rhythm disturbance than } \\
\text { euthymic patients and controls. }\end{array}$ \\
\hline $\begin{array}{l}\text { Seleem et al., } 2015 \\
\text { [49] }\end{array}$ & $\begin{array}{l}\text { Cohort study } \\
(27 \mathrm{mo})\end{array}$ & $\begin{array}{l}257 \mathrm{BD}, 105 \\
\text { non-BD and } 55 \\
\text { controls }\end{array}$ & Any & $\begin{array}{l}\text { F/M: 2.1. Mean } \\
\text { age } 43 \mathrm{y} \text {. }\end{array}$ & $\begin{array}{l}\text { CSM and sleep } \\
\text { diary }-27 \text { mo }\end{array}$ & $\begin{array}{l}\text { BD had an evening preference. BD also had a higher } \\
\text { sleep onset latency, higher wakening after sleep onset } \\
\text { and less bedtime stability and awakening time stability. }\end{array}$ \\
\hline $\begin{array}{l}\text { Cudney et al., } 2016 \\
\text { [55] }\end{array}$ & Cross-sectional & $80 \mathrm{BD}$ & $\begin{array}{l}\text { Euthymia and } \\
\text { depression }\end{array}$ & $\begin{array}{l}\text { F/M: } 4 . \text { Mean } \\
\text { age } 42.6 \mathrm{y} .\end{array}$ & BRIAN and PSQI & $\begin{array}{l}\text { Disruption in biological rhythm is associated with poor } \\
\text { quality of life in BD, independent of sleep disturbance, } \\
\text { sleep medication use, and severity of depression. }\end{array}$ \\
\hline $\begin{array}{l}\text { Faedda et al., } 2016 \\
\text { [59] }\end{array}$ & $\begin{array}{l}\text { Case-control } \\
\text { study }\end{array}$ & $\begin{array}{l}48 \mathrm{BD}, 44 \\
\text { ADHD and } 42 \\
\text { controls }\end{array}$ & Not specified & $\begin{array}{l}\text { F/M: } 1.1 \text {. } \\
\text { Children (Mean } \\
\text { age } 10.1 \mathrm{y} \text { ). }\end{array}$ & $\begin{array}{l}\text { Actigraphy }-3 \\
-5 \text { days }\end{array}$ & $\begin{array}{l}\text { BD had a reduced circadian amplitude compared to the } \\
\text { three other groups. This study confirms prior reports of } \\
\text { hyperactivity in subjects with mania or hypomania. }\end{array}$ \\
\hline $\begin{array}{l}\text { Girshkin et al., } 2016 \\
{[82]}\end{array}$ & $\begin{array}{l}\text { Case-control } \\
\text { study }\end{array}$ & $\begin{array}{l}56 \mathrm{BD}, 56 \mathrm{Sz} \\
\text { and } 59 \text { controls }\end{array}$ & Not specified & $\begin{array}{l}\text { F/M: 1.7. Mean } \\
\text { age } 37.21 \mathrm{y} \text {. }\end{array}$ & Cortisol levels & $\begin{array}{l}\text { Waking cortisol levels were greater in BD relative to Sz. } \\
\text { Lower waking cortisol levels were associated with } \\
\text { longer illness duration in BD. On the other hand, lower } \\
\text { antipsychotic dose was related to greater symptom } \\
\text { severity in Sz. }\end{array}$ \\
\hline
\end{tabular}


(17.3\%); social rhythm metric (SRM) - three studies, 234 patients (6.8\%); and morningness-eveningness questionnaire (MEQ) - four studies, 133 patients (3.9\%). 12 studies involving 356 patients (10.4\%) used objective parameters: 11 used actigraphy; and one, LifeShirt, a monitoring device in the form of a wearable upperbody garment. Three studies (129 patients, 3.8\%) measured melatonin levels; and two (121 patients, 3.5\%) evaluated daily profiles of cortisol. Only one (14 patients) examined brain function using functional magnetic resonance imaging (fMRI) (Table 1).

Mood status of patients varied widely among studies: 14 evaluated patients in euthymia (1180 patients, 34.3\%); 11 evaluated patients in any mood stage (1016 patients, 29.6\%); two, only depression (57 patients, $1.7 \%$ ); one, only mania (28 patients, $0.8 \%$ ); seven evaluated two or more mood status (341 patients, $9.9 \%$ ); and seven did not specify (814 patients, $23.7 \%$ ). Two articles assessed rapid cycling $\mathrm{BD}$ (84 patients; $2.4 \%$ ).

\section{Disruption of biological rhythm}

19 studies (45.2\%) focused on regulation of biological rhythm in BD patients and its relationships with clinical variables. All five studies (576 patients) that used subjective measures of rhythm indicated a disruption of the biological rhythm in BD patients in comparison to controls [50-55]. This was also evidenced even in drug-naïve BD patients [39]. It is suggested that disruption of biological rhythm in BD occurs independently of current mood state $(\mathrm{p}<0.001)$. Differently, the biological rhythm disruption is dependent on current mood symptoms in subjects with major depressive disorder (MDD) [52].

Four studies using actigraphy (105 patients) confirmed a less stable and more variable circadian activity patterns in $\mathrm{BD}$, even in euthymia [10,56-59]. Smaller amplitude and alterations in mean activity were demonstrated in four actigraphic studies, including pediatric samples (117 patients) [58-61].

Individuals with bipolar disorder showed longer sleep duration, greater activity in active periods of sleep and greater interday stability. Geoffroy et al., 2014 showed that the association between BD and interday stability remain significant after correction for multiple testing [61].

The link between quality of life and rhythm was poorly investigated in BD patients. Cudney et al., 2016 showed that rhythm dysregulation is associated with poor quality of life in BD, independent of sleep disturbance, sleep medication, and severity of mood symptoms. Treatment strategies targeting regulation of biological rhythms may improve quality of life in this population [55].

Few articles evaluated the bidirectional relationship between mood status and social rhythm activity. Only one prospective cohort (involving 206 patients) investigated the impact of biological rhythm on mood status. Importantly, social rhythm regularity significantly predicted the onset of major depressive, hypomanic and manic episodes [50].

Four studies (136 patients) focused on mania; all of them were case-control. Minassian et al., 2010 (28 patients) demonstrated that manic BD exhibited higher levels of motor activity when exploring novel environments [63]. Krane-Gartiser et al., 2014 (30 patients) reported lower motor activity in mania and depression compared to controls in the morning ( $p<0.001$ ), but not in the evening [64]. Mania and mixed status were associated with acrophase advance $(\mathrm{p}<0.001)$ and lower daily activity $(\mathrm{p}<0.05)$ than controls [47]. It was suggested that greater severity of manic symptoms correlated with less robust rhythms of locomotor activity [57].

Circadian disturbances were more frequent in depression phase in comparison to euthymia and controls (four studies; 260 patients) 
$[53,54,64,65]$. Even in residual depressive symptoms, this relationship was observed ( $p<0.001$ ) (66). In opposition, only one study (42 patients) found no relationship between the degree of depression and circadian quotient $(p=0.96)(57)$. Depression was associated with lower mean activity level, higher variability, delay of circadian rhythm in evening and morning activities and poor functioning $[53,54,64,65]$. Severity of mood symptoms correlated to less robust rhythms in BD, but not MDD [10].

Only one study evaluated the repercussions of psychoeducation on circadian rhythm on BD. 45 patients were divided into two groups: one group received a short-term psychoeducation model and medication (combined intervention), and the other received only medication (standard intervention). The model was summarized in a protocol of six individual sessions of one hour each about $\mathrm{BD}$, mood status and treatment. Both groups showed remission of depressive symptoms $(\mathrm{p}=0.04)$, but none showed a statistically significant reduction of manic symptoms. No influence of psychoeducation on biological rhythm was observed; however, only the standard intervention group showed improvement in the following BRIAN domains: sleep ( $p=0.01)$, activity $(\mathrm{p}<0.001)$, social rhythm $(\mathrm{p}=0.002)$ and total score $(\mathrm{p}=0.001)$ [48].

Only one study analyzed the impact of mood stabilizers on rhythm in BD. In lithium-treated patients $(n=39)$, activity levels in the evening were higher and there was a trend toward a later morning awakening during depressive episodes [66]. Other drugs were not investigated, and further studies are necessary.

A single study (18 patients) used a combined heart rate and movement registration as an indicator of rhythm alterations. It concluded that fitness, acceleration and activity energy expenditure were lower in unipolar patients, but not in the BD group (in depression or euthymia) [67].

Circadian alterations in 14 euthymic BD patients were related to structural abnormalities in the brain, mainly concerning the dorsolateral prefrontal cortex (DLPFC) and supramarginal gyri. In the right DLPFC, positive associations were found between the mean abnormal blood oxygen-dependent level (BOLD) and amplitude of circadian rhythm while an opposite effect was found in the left side [58].

\section{Chronotype}

15 studies (35.7\%) evaluated chronotype in BD patients. Bipolar disorder was often associated with eveningness. Six studies involving 850 patients showed that evening type was more common in BD as compared to controls [41,68-72]. Eveningness was maintained for a long time and therefore, it was considered a trait marker [49]. In opposition to these findings, one study (119 patients) did not evidence difference between euthymic BD and controls [73]. Chung et al., 2012 compared the chronotypes of BD and MDD patients and no difference in circadian preference was found $[74]$.

Considering the BD types, rhythm comparisons between BD-I and BD-II were scarce and showed unclear results. Baek et al., 2014 (200 patients) revealed that BD-II had higher eveningness scores than BD-I during euthymia [69]; in contrast, Chung et al., 2012 (106 patients) did not evidence differences; however, mood status was not specified [74].

Studies about rapid cycling, a particular type of BD, were few (two studies involving 84 patients). Rapid cycling BD often had less rhythmic daily routines $(\mathrm{p}=0.01)$ and completed fewer activities ( $p=0.01)$ than controls [53]. Compared to non-rapid cycling BD, they were more likely to have evening preference $(p<0.02)$ [75].

Only one study described the chronotype in 30 BD children. No differences between children with BD and controls were found.
However, BD adolescents (ages 13 y and older) endorsed greater eveningness compared to controls, similar to adults with BD [72].

Few studies $(\mathrm{n}=306)$ showed an interface between chronotype, depressive symptoms and seasonality. In general, BD-I and BD-II had a higher global seasonality score - GSS ( $p<0.028)$ and more seasonal affective disorders $-\mathrm{SAD}(\mathrm{p}<0.001)$ [69]. There was also a preference for evening $(\mathrm{p}<0.01)[67,74]$. Higher fluctuations throughout seasons were also associated with a family history for mood disorders $(p=0.035)$ [77].

Although very important, the relationship between chronotype and metabolism was rarely studied. Soreca et al., $2009(\mathrm{n}=29)$ reported that eveningness was associated with higher percentage of body fat $(\mathrm{p}=0.004)$ in euthymia, measured by a dual $\mathrm{x}$-ray absorptiometry. The CSM score was associated with an increase of $19 \%$ of the variance in percentage of body fat, independently of age, sex, 12-wk depression, mania score, and sleep quality [78].

Few studies reported the relationship between chronotype, functionality and sleep in euthymia. Evening type was related to a poor sleep quality ( $\mathrm{p}=0.01)$ in one study (67 patients) [77] and to poor functionality in BD ( $\mathrm{p}<0.001)$ in another (107 patients) [65].

Studies about the influence of chronotype on depressive symptoms showed conflicting results. Wood et al., 2009 (190 patients) reported that high depression scores are more likely to be associated with evening type [70]. On the other hand, Gonzalez et al., 2014 (42 patients) found no relationship between the degree of depression and circadian quotient [57]. Furthermore, Rosa et al., 2013 (107 patients) reported a correlation between biological rhythms with residual depressive symptoms $(\mathrm{p}<0.001)$ [65], while Giglio et al., 2010 (81 patients) indicated no association between subthreshold depressive symptoms and chronotype in BD [71]. To this date, only one investigation (81 patients) observed the influences of chronotype in mania, revealing that subthreshold manic symptoms were unrelated to chronotype [71].

\section{Circadian rhythm and biomarkers}

Six studies (14.3\%) evaluated biomarker changes throughout a day. Three of them (129 patients) measured the daily profiles of melatonin [50,79,80]; and two (121 patients) evaluated cortisol levels $[81,82]$. Another one (52 patients) analyzed lipid peroxidation, measuring malondialdehyde (MDA) levels and the activity of total and extracellular superoxide dismutase (SOD), catalase (CAT) and glutathione S-transferase (GST) [51].

Evidence indicates that daily levels of melatonin may assume a different pattern during mania as compared to controls and to patients with depression. Novakova et al., 2015 (60 patients) demonstrated that the melatonin levels in mania at 15:00 and 19:00 h were significantly higher than in controls and depression, corresponding to nearly $50 \%$ of the maximal nocturnal levels. Moreover, at 07:00 h, they were significantly lower. Otherwise, no differences were found between melatonin profiles of controls and depressive patients [80]. Melatonin secretion abnormalities were confirmed in a subgroup of euthymic BD patients: lower melatonin levels and a later peak time for melatonin was confirmed [48]. Another study (18 patients) compared melatonin levels in depressive BD and MDD groups, concluding that BD patients showed later dim light melatonin onset - DLMO (melatonin concentration above $3.000 \mathrm{pg} / \mathrm{mL}$ ) and smaller melatonin area under the curve - AUC (corresponding mean melatonin concentrations over the day) than the MDD patients. In this study, DLMO occurred after habitual sleep onset in one third of patients with mood disorders. Thereby, these findings indicate that BD patients show delayed and lower evening melatonin secretion [79].

The cortisol daily profile of $\mathrm{BD}$ patients probably presents a specific pattern. Girshkin et al., 2016 (56 patients) showed that BD 
had higher levels of cortisol at waking and increased cortisol levels in response to a stressful exam (magnetic resonance imaging MRI) than schizophrenia (but not controls). Interestingly, cortisol patterns were associated with different variables in BD and schizophrenia: lower waking cortisol indices related to longer illness duration in BD, and to greater symptom severity in schizophrenia [82]. Associations between cortisol levels and cognitive functioning or depressive symptoms were not evidenced [81].

Cudney et al., 2014 (52 patients) suggested that circadian disturbance was independently associated with increased lipid peroxidation in $\mathrm{BD}(\mathrm{p}<0.05)$, but not in controls. A reduced extracellular SOD $(\mathrm{p}<0.05)$ in BD was observed, but no differences in total SOD, CAT or GST activity was found [51]. No study correlated inflammatory cytokines and rhythm disorders in BD.

\section{Genetics}

Six studies (14.3\%) evaluated the impact of genetic alterations on circadian rhythm: two (299 patients) analyzed the CLOCK gene [64,81]; one (25 patients), acetylserotonin O-methyltransferase (ASMT) gene [60]; the fourth (8 patients) investigated polymorphisms in 15 genes: ARNTL, BHLHE40, BHLHE41, CLOCK, CRY1, CRY2, CSNK1D, CSNK1E, DBP, NFIL3, NPAS2, NR1D1, PER1, PER2, and PER3 [82]. Two studies evaluated polymorphisms in other circadian genes $[19,85]$.

Lee et al., 2010 (260 patients) studied a single nucleotide polymorphism (SNP) in the $3^{\prime}$-flanking region of CLOCK (3111T/C; rs1801260), whose 3111T/C variant showed significant allelic and genotypic associations with bipolar disorder $(\mathrm{p}=0.012$ and $\mathrm{p}=0.033$ ). Furthermore, $\mathrm{C}$ allele carriers ( $\mathrm{C} / \mathrm{C}$ and $\mathrm{C} / \mathrm{T}$ genotypes $)$ were more associated with eveningness $(p=0.041)$ [83]. Similarly, Benedetti et al., 2007 (39 patients) found that $C$ variant was associated with higher evening activity $(\mathrm{p}=0.007)$, a delayed sleep onset (mean 79 min later), and a reduced amount of sleep during the night (mean 75 min less) [66].

Geoffroy et al., 2014 (25 patients) focused on polymorphism (rs4446909) of the promoter of the ASMT gene, encoding one of the two enzymes involved in melatonin biosynthesis. An association between the GG at-risk genotype was found with each of the following variables: longer sleep duration $(p=0.03)$, greater activity during active periods of the night $(\mathrm{p}=0.015)$ and higher interday stability $(\mathrm{p}=0.003)$ [62].

Kripke et al., 2014 (8 patients) showed that delayed sleep and "eveningness" were inversely associated with loci in circadian genes NFIL3 (rs2482705) and RORC (rs3828057). Some BHLHE40 alleles were associated with non-24-h sleep-wake cycles, and less robustly, with delayed sleep and bipolar disorder (e.g., rs34883305, rs34870629, rs74439275, and rs3750275) [84].

Kripke et al., 2009 (130 patients) revealed that bipolar disorder was associated with genes NR1D1 (rs2314339), suggesting that perturbations of the circadian gene network at several levels may influence mood disorders [85]. Polymorphisms of PER3 and CSNK1E were associated with greater eveningness in BD patients.

Etain et al., 2014 (239 patients) demonstrated that rs774045 in TIMELESS was associated with eveningness whereas rs782931 in RORA was associated with rigid circadian type indicating these variants in the TIMELESS and RORA genes may confer susceptibility to BD and impact on circadian phenotypes in carriers [19].

\section{Discussion}

The vast majority of studies showed a disruption of circadian rhythm and an evening preference in BD patients, independently of mood status. Interestingly, MDD contrasts with this evidence, demonstrating that rhythm disruption is dependent on current mood symptoms [52]. Some studies showed that circadian alterations in BD were more frequent in individuals with depression than in euthymic patients $[53,54,64,65]$. Circadian disruptions were established even in drug naïve patients [52]. Actigraphic studies confirmed the disruption of the circadian rhythm [10,56-59]. Based on all this evidence, it is licit to conclude that circadian rhythm alterations are present in $\mathrm{BD}$; however, the role of mood status is unclear.

Few studies involved patients in manic state. Mania and mixed status were associated with acrophase advance and lower daily activity [61]. In addition, greater severity of manic symptoms correlated with less robust rhythms [56]. The paucity of evidence related to mania possibly relates to the obvious clinical limitations of studying these patients. This aspect needs more clarification.

Circadian disturbances may have a role in the pathogenesis of mood disorders. Robillard et al., 2013 showed that young patients with mood disorders, especially those with $\mathrm{BD}$, are likely to have a delayed sleep phase [86]. Actigraphic studies in BD patients show sleep abnormalities during depressive, manic, and interepisode periods [87]. In light of these evidences, when analyzing sleep disturbances, attention to age, circadian changes and levels of residual depressive symptoms must be taken into consideration [88].

Similarly, patients with other psychiatric disorders, such as major depression, anxiety disorders and schizophrenia often show circadian dysregulation of hormonal and behavioral processes contributing to major functional impairment [89,90]. Rhythm disruption has been associated with disease severity in some psychiatric disorders, e.g., increased suicidal ideation in patients with MDD [58].

Evidence on circadian alterations in BD is mostly based on crosssectional evaluations; therefore, a cause-effect relationship cannot be established. Interestingly, one prospective study showed that less social rhythm regularity predicted a shorter time between affective episodes [50]. These preliminary results are promising and it is fair to hypothesize that interference in the circadian rhythm could reduce affective symptoms.

One study tested the effects of psychoeducation in BD patients and suggested no significant influence on circadian changes [48]. Psychoeducation is not a conventional and recommended measure to modify circadian patterns. A change of rhythm can be achieved using measures such as light exposure, physical exercise and sleep hygiene $[20,91]$. Randomized trials focusing on circadian changes may clarify whether a long-term interference will affect the expression of mood symptoms.

One important and isolated study demonstrated effects of lithium therapy on circadian patterns in BD patients, characterized by higher evening activity and later morning awakening [66]. In vitro studies of fibroblasts from BD patients show weaker amplification of circadian rhythm by lithium and this possibly relates to calcium signaling. Possibly, a low amplitude circadian rhythm associated with lithium therapy could influence therapeutic results $[23,92]$. Genetic studies further confirm a relationship between circadian rhythm and lithium showing that the expression of circadian clock components, including Per2, Cry1 and Rev-erb $\alpha$, is affected by lithium treatment [24]. A systematic review reveals that chronic lithium treatment stabilizes activity rhythms: it delays circadian rhythms and peak of body temperature, and reduces amplitude and duration of activity rhythms [18]. Given all this evidence, clinical studies evaluating the impact of lithium and other mood stabilizers on circadian rhythm and the influence on therapeutic results are warranted.

A single study shows that circadian alterations in BD are linked to structural abnormalities in the brain mainly concerning the dorsolateral prefrontal cortex (DLPFC) and supramarginal gyri. Prefrontal dysregulation is associated with trait impulsivity and 
impairment in working memory [93,94]. Reduced white matter integrity is also observed in bipolar patients, suggesting cognitive repercussions $[95,96]$. Studies investigating alterations of functional neuroimaging in $\mathrm{BD}$ related to rhythm disruption were not found. These preliminary findings deserve confirmation.

Interestingly, one single study showed that children with $\mathrm{BD}$ did not have eveningness preference; in contrast, eveningness was already present in adolescents [72]. Recently, objective measures of sleep, circadian rhythmicity, and hyperactivity were abnormal in children with BD [59]. Reduced relative circadian amplitude was a distinctive feature. Generally, adolescents tend to sleep less, go to bed and get up later, and experience greater daytime sleepiness and weekend compensation for sleep shortage on weekdays, compared to younger children [97]. In fact, studies investigating sleep-wake patterns in preadolescents and in children are scarce $[98,99]$. Future samples should include both youth and adults with BD.

Comparisons between chronotype preference and BD types were scarce. One study showed that BD-II was more associated with eveningness. Abé et al., 2015 indicated that both BD subtypes showed cortical abnormalities, such as lower volume, thickness and surface area in frontal brain regions. However, BD-I had abnormally low cortical volume and thickness in temporal and medial prefrontal regions [100]. More studies on neuroanatomy and pathophysiology could possibly explain the differences in clinical manifestation and patterns of circadian rhythm between BD-I and II.

In $\mathrm{BD}$, few studies showed an interface between chronotype, depressive symptoms and seasonality. Preliminary evidence showed that BD-I and BD-II had a higher GSS and more seasonal affective disorders [69]. Higher fluctuations throughout seasons were also associated with a family history for mood disorders in bipolar patients [76]. The latter evidence helps to consolidate the common biological ground between mood, circadian rhythm and seasonality in BD. Recently, two rare variants in the circadian clock gene PERIOD3 (PER3-P415A/ H417R) in humans with familial advanced sleep phase and depression and seasonality were identified indicating a possible role for PER3 in mood regulation [101]. Seasonal affective disorders are frequently reported during winter in regions with reduced levels of light; however, the effects of seasonal variation on circadian rhythm, chronotype expression, sleep and mood symptoms are unclear [102].

Studies about influence of chronotype on depressive symptoms showed conflicting results. To this date, only one investigation observed the impact of circadian preference in mania, revealing that subthreshold manic symptoms were unrelated to chronotype [71]. In population based studies, eveningness has been associated with depressive symptoms [103,104]. In MDD, evening preference was related to poor therapeutic response [37,38]. Jankowski et al., 2016 suggested that morning affect may be responsible for the link between circadian preference and depressive symptoms. People with eveningness without low morning affect had less depressed/ somatic symptoms [105]. To the best of our knowledge, this issue has not been investigated in BD patients.

Despite the clinical relevance due to its frequency, morbidity and mortality, metabolic abnormalities, eating patterns and circadian variations were rarely evaluated in BD patients. Previously, eveningness was associated with higher percentage of body fat and depressive symptoms [78]. Changes in the clock system alter the neuroendocrine pathways within the hypothalamus. These structures are involved in feeding and energetics [106]. Chronically, rhythm desynchronization can enhance inflammatory mediators and may increase the risk of cardiovascular and metabolic diseases [107]. Previously, a link between a high BMI and several sleep disturbances in BD, including lower sleep efficiency has been established [108]. Given the high prevalence of these diseases, it is important to identify the impact of sleep and circadian disorders in the development and course of metabolic disease.

Considering biomarkers, preliminary evidence showed a dysregulation of daily levels of melatonin and cortisol. In relation to oxidative stress, circadian rhythm disturbance was associated with increased lipid peroxidation in BD [51]. Many authors consider BD an inflammatory condition [109-111]. To date, no study focused on the relationship between inflammatory cytokines and biological rhythms. Of note, circadian dysregulation entails changes in immune system and increase vulnerability to infections. Immune parameters also show daily variations, demonstrating a circadian pattern [112].

Currently, studies involving genetics are incipient. Many circadian genes have been associated with BD [113-115]. However, studies assessing how these genes influences circadian rhythm in $\mathrm{BD}$ are less frequent. It is suggested that chronotherapies, including sleep deprivation and sleep phase advance, modify clock gene machinery and it can represent a pathophysiological explanation to improvement of mood symptoms [116].

Several limitations need to be acknowledged. Different study designs, various kinds of circadian rhythm outcomes, and heterogeneous mood status prevented us from performing a metaanalysis. Most studies were cross-sectional, hampering a cause-effect relationship. The majority of them were based only on subjective parameters, and studies with actighaphy or biomarkers were less frequent. However, this review included 3432 patients with BD evaluating aspects related to chronotype, circadian disruption, disease severity, biomarkers, genetic influences and therapeutic aspects. The evidence clarifies some issues and gives avenues to new investigations.

In conclusion, circadian rhythm disruption and eveningness are common in BD. However, the impact on mood status is still unclear More prospective research is needed. Future studies should focus on therapy, outcomes, metabolic alterations, rapid cycling, biomarkers variability, children's evaluation, and brain structural abnormalities. Longitudinal data that assess when/how circadian preference/rhythms change, and how these changes relate to age of diagnosis and symptom severity could expand the knowledge about the subject.

\section{Practice points}

1) Disruption of circadian rhythm was identified, even in drug-naïve BD patients. Actigraphy confirmed this finding, independently of mood status. Studies showing alterations in daily profiles of melatonin levels and cortisol reinforced circadian dysregulations in BD.

2) Evening type was more common in BD adults. It was suggested that children did not show eveningness; however, this circadian preference was already found in adolescents. The influence of chronotype on mood symptoms remains unclear.

3) Genetic studies involving circadian rhythm are incipient. Analysis of the circadian genes suggested that eveningness is more associated with $\mathrm{C}$ allele carriers in CLOCK genes and inversely related to loci in circadian genes NFIL3 (rs2482705) and RORC (rs3828057). 


\section{Research agenda}

Areas for future research may include:

1) Repercussions of circadian disruption on mood symptoms.

2) Influence of morning affect on chronotype and mood symptoms.

3) Relationships between metabolic abnormalities, eating patterns and circadian variations.

4) Profile of the circadian rhythm in children, adolescents and adults with BD.

5) Relationship between rapid cycling, chronotype and biological rhythm.

6) Effects of mood stabilizers on circadian patterns.

7) Associations between neuroanatomy, inflammatory mediators and biological rhythm.

\section{Conflicts of interest}

The authors do not have any conflicts of interest to disclose.

\section{References}

[1] Schnell A, Albrecht U, Sandrelli F. Rhythm and mood: relationships between the circadian clock and mood-related behavior. Behav Neurosci 2014; $128: 326-43$.

[2] Cretu JB, Culver JL, Goffin KC, Shah S, Ketter TA. Sleep, residual mood symptoms, and time to relapse in recovered patients with bipolar disorder. J Affect Disord 2016;190:162-6.

[3] Vitale JA, Roveda E, Montaruli A, Galasso L, Weydahl A, Caumo A, et al, Chronotype influences activity circadian rhythm and sleep: differences in sleep quality between weekdays and weekend. Chronobiol Int 2015;32(3):405-15.

[4] Diaz-Morales JF, Escribano C, Jankowski KS. Chronotype and time-of-day effects on mood during school day. Chronobiol Int 2015;32(1):37-42.

[5] Gonçalves BS, Cavalcanti PR, Tavares GR, Campos TF, Araujo JF. Nonparametric methods in actigraphy: an update. Sleep Sci 2014;7(3):158-64.

[6] McClung CA. How might circadian rhythms control mood? Let me count the ways. Biol Psychiatry 2013;74:242-9.

[7] Hirata FC, Lima MC, de Bruin VM, Nobrega PR, Wenceslau GP, de Bruin PF. Depression in medical school: the influence of morningness-eveningness. Chronobiol Int 2007;24:939-46.

[8] Logan RW, McClung CA. Animal models of bipolar mania: the past, present and future. Neuroscience 2016;321:163-88.

[9] Arent CO, Valvassori SS, Steckert AV, Resende WR, Dal-Pont GC, LopesBorges J, et al. The effects of n-acetylcysteine and/or deferoxamine on manic-like behavior and brain oxidative damage in mice submitted to the paradoxal sleep deprivation model of mania. J Psychiatr Res 2015;65:71-9.

[10] Grierson AB, Hickie IB, Naismith SL, Hermens DF, Scott EM, Scott J. Circadian rhythmicity in emerging mood disorders: state or trait marker? Int J Bipolar Disord 2016;4:3.

[11] Bechtel W. Circadian rhythms and mood disorders: are the phenomena and mechanisms causally related? Front Psychiatry 2015;6:118.

[12] Malhi GS, Kuiper S. Chronobiology of mood disorders. Acta Psychiatr Scand Suppl 2013;444:2-15.

[13] Kanady JC, Soehnera AM, Harvey AG. A retrospective examination of sleep disturbance across the course of bipolar disorder. J Sleep Disord Ther 2015;4:1000193.

[14] Stange JP, Kleiman EM, Sylvia LG, Magalhaes PV, Berk M, Nierenberg AA et al. Specific mood symptoms confer risk for subsequent suicidal ideation in bipolar disorder with and without suicide attempt history: multi-wave data from step-BD. Depress Anxiety 2016;33:464-72.

*[15] Ng TH, Chung KF, Ho FY, Yeung WF, Yung KP, Lam TH. Sleep-wake disturbance in interepisode bipolar disorder and high-risk individuals: a systematic review and meta-analysis. Sleep Med Rev 2015;20:46-58.

[16] Soreca I. Circadian rhythms and sleep in bipolar disorder: implications for pathophysiology and treatment. Curr Opin Psychiatry 2014;27:467-71.

[17] Gonzalez R. The relationship between bipolar disorder and biological rhythms. J Clin Psychiatry 2014;75:e323-31.

\footnotetext{
* The most important references are denoted by an asterisk.
}

[18] Moreira J, Geoffroy PA. Lithium and bipolar disorder: impacts from molecular to behavioural circadian rhythms. Chronobiol Int 2016:33:351-73.

[19] Etain B, Jamain S, Milhiet V, Lajnef M, Boudebesse C, Dumaine A, et al. Association between circadian genes, bipolar disorders and chronotypes. Chronobiol Int 2014;31(7):807-14.

*[20] Abreu T, Braganca M. The bipolarity of light and dark: a review on bipolar disorder and circadian cycles. J Affect Disord 2015;185:219-29.

[21] Rybakowski JK, Dmitrzak-Weglarz M, Dembinska-Krajewska D, Hauser J, Akiskal KK, Akiskal HH. Polymorphism of circadian clock genes and temperamental dimensions of the TEMPS-A in bipolar disorder. J Affect Disord 2014;159:80-4.

[22] Dmitrzak-Weglarz MP, Pawlak JM, Maciukiewicz M, Moczko J, Wilkosc M, Leszczynska-Rodziewicz A, et al. Clock gene variants differentiate mood disorders. Mol Biol Rep 2015;42:277-88.

[23] McCarthy MJ, Le Roux MJ, Wei H, Beesley S, Kelsoe JR, Welsh DK. Calcium channel genes associated with bipolar disorder modulate lithium's amplification of circadian rhythms. Neuropharmacology 2016;101:439-48.

[24] Schnell A, Sandrelli F, Ranc V, Ripperger JA, Brai E, Alberi L, et al. Mice lacking circadian clock components display different mood-related behaviors and do not respond uniformly to chronic lithium treatment. Chronobiol Int 2015;32:1075-89.

[25] Poon SH, Sim K, Sum MY, Kuswanto CN, Baldessarini RJ. Evidence-based options for treatment-resistant adult bipolar disorder patients. Bipolar Disord 2012;14:573-84.

[26] Henriksen TE, Skrede S, Fasmer OB, Hamre B, Gronli J, Lund A. Blocking blue light during mania - markedly increased regularity of sleep and rapid improvement of symptoms: a case report. Bipolar Disord 2014;16: 894-8.

[27] Crowe M, Beaglehole B, Inder M. Social rhythm interventions for bipolar disorder: a systematic review and rationale for practice. J Psychiatr Ment Health Nurs 2016:23:3-11.

[28] Tseng PT, Chen YW, Tu KY, Chung W, Wang HY, Wu CK, et al. Light therapy in the treatment of patients with bipolar depression: a meta-analytic study. Eur Neuropsychopharmacol 2016:26:1037-47.

[29] Yu JH, Yun CH, Ahn JH, Suh S, Cho HJ, Lee SK, et al. Evening chronotype is associated with metabolic disorders and body composition in middleaged adults. J Clin Endocrinol Metab 2015;100:1494-502.

[30] Friborg O, Rosenvinge JH, Wynn R, Gradisar M. Sleep timing, chronotype, mood, and behavior at an Arctic latitude (69 degrees N). Sleep Med 2014; $15: 798-807$.

[31] Juda M, Vetter C, Roenneberg T. Chronotype modulates sleep duration, sleep quality, and social jet lag in shift-workers. J Biol Rhythms 2013;28: $141-51$.

[32] Miller MA, Rothenberger SD, Hasler BP, Donofry SD, Wong PM, Manuck SB, et al. Chronotype predicts positive affect rhythms measured by ecological momentary assessment. Chronobiol Int 2015;32:376-84.

[33] Simor P, Zavecz Z, Palosi V, Torok C, Koteles F. The influence of sleep complaints on the association between chronotype and negative emotionality in young adults. Chronobiol Int 2015;32:1-10.

[34] Fares S, Hermens DF, Naismith SL, White D, Hickie IB, Robillard R. Clinical correlates of chronotypes in young persons with mental disorders. Chronobiol Int 2015:32:1183-91.

[35] Muller MJ, Kundermann B, Cabanel N. Eveningness and poor sleep quality independently contribute to self-reported depression severity in psychiatric inpatients with affective disorder. Nord J Psychiatry 2016;70:329-34.

[36] Bahk YC, Han E, Lee SH. Biological rhythm differences and suicidal ideation in patients with major depressive disorder. J Affect Disord 2014;168: 294-7.

[37] Chan JW, Lam SP, Li SX, Yu MW, Chan NY, Zhang J, et al. Eveningness and insomnia: independent risk factors of nonremission in major depressive disorder. Sleep 2014;37:911-7.

[38] Corruble E, Frank E, Gressier F, Courtet P, Bayle F, Llorca PM, et al Morningness-eveningness and treatment response in major depressive disorder. Chronobiol Int 2014;31:283-9.

[39] Shawa N, Roden LC. Chronotype of South African adults is affected by solar entrainment. Chronobiol Int 2016:33(3):315-23.

[40] Boudebesse C, Geoffroy PA, Bellivier F, Henry C, Folkard S, Leboyer M, et al. Correlations between objective and subjective sleep and circadian markers in remitted patients with bipolar disorder. Chronobiol Int 2014;31:698-704.

[41] Boudebesse C, Lajnef M, Geoffroy PA, Bellivier F, Nieto I, Gard S, et al. Chronotypes of bipolar patients in remission: validation of the French version of the circadian type inventory in the FACE-BD sample. Chronobiol Int 2013;30:1042-9.

[42] Adan A, Archer SN, Hidalgo MP, Di Milia L, Natale V, Randler C. Circadian typology: a comprehensive review. Chronobiol Int 2012;29(9):1153-75.

[43] Caci H, Nadalet L, Staccini P, Myquel M, Boyer P. The composite scale of morningness: further psychometric properties and temporal stability. Eur Psychiatry 2000;15(4):278-81.

[44] Giglio LM, Magalhaes PV, Andreazza AC, Walz JC, Jakobson L, Rucci P, et al Development and use of a biological rhythm interview. J Affect Disord 2009;118(1-3):161-5.

[45] Kaplan KA, Talbot LS, Gruber J, Harvey AG. Evaluating sleep in bipolar disorder: comparison between actigraphy, polysomnography, and sleep diary. Bipolar Disord 2012;14(8):870-9. 
[46] Chan S, Debono M. Replication of cortisol circadian rhythm: new advances in hydrocortisone replacement therapy. Ther Adv Endocrinol Metab 2010;1(3):129-38.

[47] Faria AD, de Mattos Souza LD, de Azevedo Cardoso T, Pinheiro KA, Pinheiro RT, da Silva RA, et al. The influence of psychoeducation on regulating biological rhythm in a sample of patients with bipolar II disorder: a randomized clinical trial. Psychol Res Behav Manag 2014;7: $167-74$.

[48] Nurnberger Jr JI, Adkins S, Lahiri DK, Mayeda A, Hu K, Lewy A, et al. Melatonin suppression by light in euthymic bipolar and unipolar patients. Arch Gen Psychiatry 2000;57(6):572-9.

*[49] Seleem MA, Merranko JA, Goldstein TR, Goldstein BI, Axelson DA, Brent DA, et al. The longitudinal course of sleep timing and circadian preferences in adults with bipolar disorder. Bipolar Disord 2015;17: 392-402.

*[50] Shen GH, Alloy LB, Abramson LY, Sylvia LG. Social rhythm regularity and the onset of affective episodes in bipolar spectrum individuals. Bipolar Disord 2008; 10:520-9.

[51] Cudney LE, Sassi RB, Behr GA, Streiner DL, Minuzzi L, Moreira JC, et al. Alterations in circadian rhythms are associated with increased lipid peroxidation in females with bipolar disorder. Int J Neuropsychopharmacol 2014; $17: 715-22$.

*[52] Duarte Faria A, Cardoso TA, Campos Mondin T, Souza LD, Magalhaes PV, Patrick Zeni C, et al. Biological rhythms in bipolar and depressive disorders: a community study with drug-naive young adults. J Affect Disord 2015;186:145-8.

[53] Ashman SB, Monk TH, Kupfer DJ, Clark CH, Myers FS, Frank E, et al. Relationship between social rhythms and mood in patients with rapid cycling bipolar disorder. Psychiatry Res 1999;86:1-8.

*[54] Pinho M, Sehmbi M, Cudney LE, Kauer-Sant'anna M, Magalhaes PV, Reinares $\mathrm{M}$, et al. The association between biological rhythms, depression, and functioning in bipolar disorder: a large multi-center study. Acta Psychiatr Scand 2016;133:102-8.

[55] Cudney LE, Frey BN, Streiner DL, Minuzzi L, Sassi RB. Biological rhythms are independently associated with quality of life in bipolar disorder. Int J Bipolar Disord 2016;4(1):9.

[56] Gonzalez R, Tamminga CA, Tohen M, Suppes T. The relationship between affective state and the rhythmicity of activity in bipolar disorder. J Clin Psychiatry 2014;75:e317-22.

[57] Jones SH, Hare DJ, Evershed K. Actigraphic assessment of circadian activity and sleep patterns in bipolar disorder. Bipolar Disord 2005;7:176-86.

*[58] McKenna BS, Drummond SP, Eyler LT. Associations between circadian activity rhythms and functional brain abnormalities among euthymic bipolar patients: a preliminary study. J Affect Disord 2014;164:101-6.

[59] Faedda GL, Ohashi K, Hernandez M, McGreenery CE, Grant MC, Baroni A et al. Actigraph measures discriminate pediatric bipolar disorder from attention-deficit/hyperactivity disorder and typically developing controls. J Child Psychol Psychiatry 2016:57:706-16.

[60] Rock P, Goodwin G, Harmer C, Wulff K. Daily rest-activity patterns in the bipolar phenotype: a controlled actigraphy study. Chronobiol Int 2014;31: 290-6.

[61] Salvatore P, Ghidini S, Zita G, De Panfilis C, Lambertino S, Maggini C, et al. Circadian activity rhythm abnormalities in ill and recovered bipolar I disorder patients. Bipolar Disord 2008;10:256-65.

[62] Geoffroy PA, Boudebesse C, Henrion A, Jamain S, Henry C, Leboyer M, et al. An ASMT variant associated with bipolar disorder influences sleep and circadian rhythms: a pilot study. Genes Brain Behav 2014;13:299-304.

[63] Minassian A, Henry BL, Geyer MA, Paulus MP, Young JW, Perry W. The quantitative assessment of motor activity in mania and schizophrenia. J Affect Disord 2010;120:200-6.

[64] Krane-Gartiser K, Henriksen TE, Morken G, Vaaler A, Fasmer OB. Actigraphic assessment of motor activity in acutely admitted inpatients with bipolar disorder. PloS One 2014;9:e89574.

[65] Rosa AR, Comes M, Torrent C, Sole B, Reinares M, Pachiarotti I, et al. Biological rhythm disturbance in remitted bipolar patients. Int J Bipolar Disord 2013;1:6.

[66] Benedetti F, Dallaspezia S, Fulgosi MC, Lorenzi C, Serretti A, Barbini B, et al. Actimetric evidence that CLOCK 3111 T/C SNP influences sleep and activity patterns in patients affected by bipolar depression. Am J Med Genet 2007; 144:631-5.

[67] Faurholt-Jepsen M, Brage S, Vinberg M, Christensen EM, Knorr U, Jensen HM, et al. Differences in psychomotor activity in patients suffering from unipolar and bipolar affective disorder in the remitted or mild/ moderate depressive state. J Affect Disord 2012;141:457-63.

[68] Ahn YM, Chang J, Joo YH, Kim SC, Lee KY, Kim YS. Chronotype distribution in bipolar I disorder and schizophrenia in a Korean sample. Bipolar Disord 2008; $10: 271-5$.

[69] Baek JH, Kim JS, Kim MJ, Ryu S, Lee K, Ha K, et al. Lifetime characteristics of evening-preference and irregular bed-rise time are associated with lifetime seasonal variation of mood and behavior: comparison between individuals with bipolar disorder and healthy controls. Behav Sleep Med 2016;14:155-68.

[70] Wood J, Birmaher B, Axelson D, Ehmann M, Kalas C, Monk K, et al. Replicable differences in preferred circadian phase between bipolar disorder patients and control individuals. Psychiatry Res 2009;166:201-9.
[71] Giglio LM, Magalhaes PV, Andersen ML, Walz JC, Jakobson L, Kapczinski F. Circadian preference in bipolar disorder. Sleep Breath 2010;14:153-5.

*[72] Kim KL, Weissman AB, Puzia ME, Cushman GK, Seymour KE, Wegbreit E et al. Circadian phase preference in pediatric bipolar disorder. J Clin Med Res 2014;3:255-66.

[73] Saunders EF, Novick DM, Fernandez-Mendoza J, Kamali M, Ryan KA Langenecker SA, et al. Sleep quality during euthymia in bipolar disorder: the role of clinical features, personality traits, and stressful life events. Int ] Bipolar Disord 2013:1:16.

[74] Chung JK, Lee KY, Kim SH, Kim EJ, Jeong SH, Jung HY, et al. Circadian rhythm characteristics in mood disorders: comparison among bipolar disorder I, bipolar disorder II and recurrent major depressive disorder. Clin Psychopharmacol Neurosci 2012;10:110-6.

[75] Mansour HA, Wood J, Chowdari KV, Dayal M, Thase ME, Kupfer DJ, et al. Circadian phase variation in bipolar I disorder. Chronobiol Int 2005;22: $571-84$.

[76] Hakkarainen R, Johansson C, Kieseppa T, Partonen T, Koskenvuo M Kaprio J, et al. Seasonal changes, sleep length and circadian preference among twins with bipolar disorder. BMC Psychiatry 2003;3:6.

[77] Brambilla C, Gavinelli C, Delmonte D, Fulgosi MC, Barbini B, Colombo C, et al. Seasonality and sleep: a clinical study on euthymic mood disorder patients. Depress Res Treat 2012;2012:978962.

*[78] Soreca I, Fagiolini A, Frank E, Goodpaster BH, Kupfer DJ. Chronotype and body composition in bipolar disorder. Chronobiol Int 2009;26:780-8.

[79] Robillard R, Naismith SL, Rogers NL, Scott EM, Ip TK, Hermens DF, et al Sleep-wake cycle and melatonin rhythms in adolescents and young adults with mood disorders: comparison of unipolar and bipolar phenotypes. Eur Psychiatry 2013;28:412-6.

*[80] Novakova M, Prasko J, Latalova K, Sladek M, Sumova A. The circadian system of patients with bipolar disorder differs in episodes of mania and depression. Bipolar Disord 2015;17:303-14.

[81] van der Werf-Eldering MJ, Riemersma-van der Lek RF, Burger $H$ Holthausen EA, Aleman A, Nolen WA. Can variation in hypothalamicpituitary-adrenal (HPA)-axis activity explain the relationship between depression and cognition in bipolar patients? PloS One 2012;7:e37119.

[82] Girshkin L, O'Reilly N, Quide Y, Teroganova N, Rowland JE, Schofield PR et al. Diurnal cortisol variation and cortisol response to an MRI stressor in schizophrenia and bipolar disorder. Psychoneuroendocrinology 2016;67: $61-9$.

[83] Lee KY, Song JY, Kim SH, Kim SC, Joo EJ, Ahn YM, et al. Association between CLOCK 3111T/C and preferred circadian phase in Korean patients with bipolar disorder. Prog Neuropsychopharmacol Biol Psychiatry 2010;34: 1196-201.

[84] Kripke DF, Klimecki WT, Nievergelt CM, Rex KM, Murray SS, Shekhtman T, et al. Circadian polymorphisms in night owls, in bipolars, and in non-24 hour sleep cycles. Psychiatry Investig 2014;11:345-62.

[85] Kripke DF, Nievergelt CM, Joo EJ, Shekhtman T, Kelsoe JR. Circadian polymorphisms associated with affective disorders. J Circadian Rhythms 2009;7:2.

[86] Robillard R, Naismith SL, Rogers NL, Ip TKC, Hermens DF, Scott EM, et al. Delayed sleep phase in young people with unipolar or bipolar affective disorders. J Affect Disord 2013;145:260-3.

[87] Geoffroy PA, Boudebesse C, Bellivier F, Lajnef M, Henry C, Leboyer M, et al. Sleep in remitted bipolar disorder: a naturalistic case-control study using actigraphy. J Affect Disord 2014:158:1-7.

[88] Geoffroy PA, Scott J, Boudebesse C, Lajnef M, Henry C, Leboyer M, et al Sleep in patients with remitted bipolar disorder: a meta anlysis of actigrafic studies. Acta Psychiatr Scand 2015;131(2):89-99.

[89] Jones SG, Benca RM. Circadian disruption in psychiatric disorders. Sleep Med Clin 2015; 10:481-93.

[90] Asarnow LD, Soehner AM, Harvey AG. Circadian rhythms and psychiatric illness. Curr Opin Psychiatry 2013:26:566-71.

[91] Melo MC, Daher EF, Albuquerque SG, de Bruin VM. Exercise in bipolar patients: a systematic review. J Affect Disord 2016;198:32-8.

[92] Noguchi T, Lo K, Diemer T, Welsh DK. Lithium effects on circadian rhythms in fibroblasts and suprachiasmatic nucleus slices from Cry knockout mice. Neurosci Lett 2016;619:49-53.

[93] Dell'Osso B, Cinnante C, Di Giorgio A, Cremaschi L, Palazzo MC Cristoffanini M, et al. Altered prefrontal cortex activity during working memory task in bipolar disorder: a functional magnetic resonance imaging study in euthymic bipolar I and II patients. J Affect Disord 2015;184 $116-22$.

[94] Mason L, O'Sullivan N, Montaldi D, Bentall RP, El-Deredy W. Decisionmaking and trait impulsivity in bipolar disorder are associated with reduced prefrontal regulation of striatal reward valuation. Brain 2014;137 2346-55.

[95] Bauer IE, Ouyang A, Mwangi B, Sanches M, Zunta-Soares GB, Keefe RS, et al. Reduced white matter integrity and verbal fluency impairment in young adults with bipolar disorder: a diffusion tensor imaging study. J Psychiatr Res 2015;62:115-22.

[96] Marlinge E, Bellivier F, Houenou J. White matter alterations in bipolar disorder: potential for drug discovery and development. Bipolar Disord 2014:16:97-112.

[97] Gau SS, Merikangas KR. Similarities and differences in sleep-wake patterns among adults and their children. Sleep 2004;27(2):299-304. 
[98] Russo PM, Bruni O, Lucidi F, Ferri R, Violani C. Sleep habits and circadian preference in Italian children and adolescents. J Sleep Res 2007;16(2): $163-9$.

[99] Giannotti F, Cortesi F, Sebastiani T, Vagnoni C. Sleeping habits in Italian children and adolescents. Sleep Biol Rhythms 2005;3(1):15-21.

[100] Abé C, Ekman CJ, Sellgren C, Petrovic P, Ingvar M, Landen M. Cortical thickness, volume and surface area in patients with bipolar disorder types I and II. J Psychiatry Neurosci 2015;41:150093.

[101] Zhang L, Hirano A, Hsu PK, Jones CR, Sakai N, Okuro M, et al. A PERIOD3 variant causes a circadian phenotype and is associated with a seasonal mood trait. Proc Natl Acad Sci U S A 2016;113:e1536-44.

[102] O'Hare C, O'Sullivan V, Flood S, Kenny RA. Seasonal and meteorological associations with depressive symptoms in older adults: a geoepidemiological study. J Affect Disord 2016;191:172-9.

[103] Merikanto I, Suvisaari J, Lahti T, Partonen T. Eveningness relates to burnout and seasonal sleep and mood problems among young adults. Nord J Psychiatry 2016;70(1):72-80.

[104] Merikanto I, Kronholm E, Peltonen M, Laatikainen T, Vartiainen E, Partonen T. Circadian preference links to depression in general adult population. J Affect Disord 2015;188:143-8.

[105] Jankowski KS. Morningness-eveningness and depressive symptoms: test on the components level with CES-D in Polish students. J Affect Disord 2016;196:47-53.

[106] Maury E, Hong HK, Bass J. Circadian disruption in the pathogenesis of metabolic syndrome. Diabetes Metab 2014;40:338-46.

[107] Machado RM, Koike MK. Circadian rhythm, sleep pattern, and metabolic consequences: an overview on cardiovascular risk factors. Horm Mol Biol Clin Investig 2014;18(1):47-52.
[108] Boudebesse C, Geoffoy PA, Henry C, Germain A, Scott J, Lajnef M, et al. Links between sleep and body mass index in bipolar disorders: an exploratory study. Eur Psychiatry 2015;30(1):89-93.

[109] Muneer A. Bipolar disorder: role of inflammation and the development of disease biomarkers. Psychiatry Investig 2016;13:18-33.

[110] Anderson G, Maes M. Bipolar disorder: role of immune-inflammatory cytokines, oxidative and nitrosative stress and tryptophan catabolites. Curr Psychiatry Rep 2015;17:8.

[111] Bauer IE, Pascoe MC, Wollenhaupt-Aguiar B, Kapczinski F, Soares JC. Inflammatory mediators of cognitive impairment in bipolar disorder. J Psychiatr Res 2014;56:18-27.

[112] Voigt RM, Forsyth CB, Keshavarzian A. Circadian disruption: potential implications in inflammatory and metabolic diseases associated with alcohol. Alcohol Res 2013;35:87-96.

[113] Gonzalez R, Gonzalez S, Villa E, Ramirez M, Zavala J, Armas R, et al. Identification of circadian gene variants in bipolar disorder in Latino populations. J Affect Disord 2015;186:367-75.

[114] Pawlak J, Dmitrzak-Weglarz M, Maciukiewicz M, Wilkosc M, LeszczynskaRodziewicz A, Zaremba D, et al. Suicidal behavior in the context of disrupted rhythmicity in bipolar disorder-data from an association study of suicide attempts with clock genes. Psychiatry Res 2015;226:517-20.

[115] Mansour HA, Talkowski ME, Wood J, Chowdari KV, McClain L, Prasad K, et al. Association study of 21 circadian genes with bipolar I disorder, schizoaffective disorder, and schizophrenia. Bipolar Disord 2009;11:701-10.

[116] Bunney BG, Bunney WE. Mechanisms of rapid antidepressant effects of sleep deprivation therapy: clock genes and circadian rhythms. Biol Psychiatry 2013;73:1164-71. 